•综述・

\title{
模式动物在共生微生物研究中的作用
}

\author{
肖雅倩 ${ }^{1}$ 刘 传 ${ }^{2}$ 肖 亮 $^{2^{*}}$ \\ 1 (北京师范大学水科学研究院, 北京 100875) \\ 2 (深圳华大生命科学研究院宏基因组研究中心, 广东深圳 518000)
}

\begin{abstract}
摘要: 共生微生物是一类定殖于宿主体表或体内, 可执行宿主本身无法完成的功能, 并依赖于宿主所提供的生长 环境的微生物。众多研究表明, 人体肠道共生微生物与免疫、营养、代谢, 甚至精神健康等生理功能密切相关, 是 重要的“微生物器官”。在早期的肠道微生物研究中, 模式动物就已经作为研究工具被使用。随着肠道微生物研究 的不断深入, 模式动物作为不可替代的研究对象发挥了越来越重要的作用。本综述主要对几种重要的模式动物如 斑马鱼(Danio rerio)、小鼠(Mus musculus)、猪(Sus scrofa domesticus)和猕猴(Macaca mulatta) 在肠道微生物研究中 的应用进行了总结, 介绍了各种模式动物的发展过程及特点, 各自在应用于研究时的优缺点, 以及利用这些动物 模型在共生微生物领域所取得的一些标志性的科研成果。同时, 也就近年来在共生微生物领域新兴的一些模式生 物如蜜蜂(Apis)、果蝇(Drosophila)、秀丽隐杆线虫(Caenorhabditis elegans)等进行了一些探讨。旨在让该领域的研 究者们了解模式动物与人体在共生微生物方面的异同, 为更好地利用这一研究工具提供参考。
\end{abstract}

关键词: 共生微生物; 宿主; 模式动物; 肠道微生物

\section{The role of model animals in the study of symbiotic microorganisms}

\author{
Yaqian Xiao ${ }^{1}$, Chuan $\mathrm{Liu}^{2}$, Liang Xiao ${ }^{2 *}$
}

1 College of Water Sciences, Beijing Normal University, Beijing 100875

2 BGI-Shenzhen, Shenzhen, Guangdong 518000

\begin{abstract}
Symbiotic microorganisms colonize external or internal surfaces of a host depending on environmental factors, and may supply the host with special functions. More and more researchers have proven that symbiotic gut microorganisms are related to a diverse range of physiological functions of a host including immunity, nutrition, metabolism and even mental health. Thus, gut microorganisms comprise an important "microbial organ" in humans. Since the early days of microbiota research, animal models have been used frequently for their microbiota, contributing greatly to new research in this field. This review provides an overview of animals used as models in symbiotic microorganism studies, including zebrafish (Danio rerio), mice (Mus musculus), pigs (Sus scrofa domesticus), and monkeys (Macaca mulatta). We provide insight into the development and characteristics of these model animals, highlighting the advantages and disadvantages of each model, as well as any outstanding scientific achievements based on their use. We also note that honey bee (Apis), fruit fly (Drosophila) and nematode (Caenorhabditis elegans) models are emerging as more prevalent in recent gut microbiota studies. This paper will contribute to better understanding the similarities and differences between the microbiota of model animals and humans, while providing useful information for effectively implementing these animal models in future research.
\end{abstract}

Key words: symbiotic microorganisms; host; animal models; gut microbiota

共生是两种生物彼此互利地生存在一起, 缺此 少彼都不能生存的一类种间关系, 是生物之间相互 关系的高度发展。豆科植物和固氮细菌之间即是典 型的植物和微生物之间的共生关系。动物和微生物
共生的案例也有很多, 如牛、羊等反刍动物与瘤胃 微生物的共生就是其中之一。随着对人体内环境认 识的发展, 人体和肠道菌群之间的共生关系也逐渐 被了解。一般情况下, 人体肠道正常菌群的巨大数 
量可以阻止和抑制外来微生物的入侵, 从而抵御某 些病原微生物的感染。此外, 它们还能提供人体自 身不能合成的维生素B1、B2、K及叶酸等营养物质 (Ramakrishna, 2013; Rowland et al, 2018), 而人体肠 道则为这些微生物提供了良好的栖息场所和生长 环境。因此, 共生微生物可以被定义为一类定殖于 宿主体表或体内，可执行宿主本身无法完成的功能， 并依赖于宿主所提供的生长环境的微生物。

随着宏基因组测序技术的发展，我们已经可以 从给定的环境样本中获取所存在的全部微生物的 遗传信息。人体微生物特别是人体肠道微生物, 其 基因数目远超宿主本身, 并且因为其在宿主代谢和 免疫调节方面发挥的重要作用(Clemente et al, 2012; Sommer \& Bäckhed, 2013; Marchesi et al, 2016)而备 受研究者关注。越来越多的研究报道揭示了菌群和 人类疾病之间的关联, 表明肠道菌群的紊乱极有可 能是多种疾病的关键风险因素(Xie et al, 2016; Berer et al, 2017; Jie et al, 2017; Liu et al, 2017)。随 着研究领域的不断扩展, 研究者的眼光也不仅仅局 限于人体肠道微生物, 皮肤、口腔、生殖道等环境 中的微生物信息及其与宿主健康之间的关联也已 经被报道(Al-Shehri et al, 2016; Kilian et al, 2016; Zákostelská et al, 2016; Haque et al, 2017)。越来越多 的研究表明, 人体各个环境的共生微生物对人体健康 有着重要影响, 与众多疾病的发生发展密切相关。

随着宏基因组研究的进展, 发展出了一套较为 成熟的研究方法, 即宏基因组关联分析 (metagenome-wide association) (Qin et al, 2012; Wang \& Jia, 2016), 并已应用于众多科研项目中。该方法可以解 析出与疾病状态或各种相关指标存在关联的微生 物基因、功能通路及特定微生物物种信息。随着宏 基因组领域研究的不断深入, 研究者们不再满足于 “关联”研究, 进而开始探索人体共生微生物与宿主 健康状态之间的因果关系及互作机理, 这将为人体 共生微生物在医疗保健及临床干预方面的应用奠 定理论基础。而这一深入探索首先在研究对象上遇 到了瓶颈。在“关联”分析中, 研究对象通常是人群, 将来自不同人群的样本的宏基因组信息进行比对, 找出差异及其与表型数据的关联。但由于不同环境 因素(人种、居住地、饮食习惯、工作环境等)造成 个体间共生微生物组成的巨大差异, 研究者们通常 需要较大量的样本才能在分析中排除数据 “噪音”,
得出可靠的分析结果。而解析“因果”关系，不但同 样需要较大的人群样本, 还需要对人群进行组织和 跟踪随访工作, 在成本和研究周期方面要求很高, 同时，对个体表型信息的跟踪、收集也有更高的要 求。最为重要的一点，在正常的伦理限制下，“科赫 法则”完全无法在以人为研究对象的科研中实现。 因此, 对于人体共生微生物的深入研究来说, 人群 本身并不是适合的研究对象。

动物模型在各个科学研究领域都扮演着重要 角色。同样，在人体共生微生物领域，模式动物因 其高度一致性的背景、可控的实验环境及可人工干 预的特点, 发挥着重要作用。特别是对于着眼于解 析“因果”关系的研究者们来说，符合动物伦理的实 验动物是深入研究共生微生物与宿主间相互作用 的最佳研究对象和重要研究工具。在本综述中, 我 们将对宏基因组领域常见或不太常见的动物模型 及相关的经典研究报道进行简析, 展现动物模型在 宏基因组研究中的关键作用，并讨论其优劣势，以 便于研究者们更好地了解和运用动物模型辅助科 学研究。

\section{1 几种重要的模式动物在共生微生物研 应用}

\section{1 斑马鱼(Danio rerio)模型}

斑马鱼是一种热带淡水鱼，因其体侧具有像斑 马一样纵向的暗蓝色与银色相间的条纹而得名。 2013年4月，英国桑格中心(Sanger Centre)协同全球 最权威的14家斑马鱼研究机构, 将斑马鱼全基因组 和人类基因组进行比较分析发现，斑马鱼基因组含 有的26,206个蛋白编码基因与人类基因高度同源, 人类 2 万多个蛋白编码基因都至少可以在斑马鱼体 内找到1个同源基因与之对应(Howe et al, 2013)。其 独特的生物学、基因组学、遗传学优势及其高度保 守的疾病信号传导路径, 使其成为研究人类疾病信 号传导途径及活体高通量药物篎选的最佳模式生 物之一。

而早在斑马鱼自身的基因组被解析之前, 研究 者们已经开始意识到这一模式动物在肠道菌群研 究方面的作用。Rawls等(2004)报道了无菌(Germ Free, GF)斑马鱼技术, 首次将斑马鱼这一模式动物 引入了菌群研究领域。研究者们将无菌斑马鱼消化 道的基因表达情况跟常规斑马鱼进行了比较, 发现 
有超过200个基因受到肠道微生物调控; 同时将其 与小鼠的消化道基因进行比较, 发现59个基因的表 达情况在这两个物种中是保守的。这一研究成果为 斑马鱼在宿主一肠道微生物互作研究中的应用奠定 了科学基础。此外, Keller等(2008)建立的数字扫描 激光光谱荧光显微技术, 可以实现对体内细胞的直 接观察, 虽然该技术最初的应用是观察斑马鱼的胚 胎发育过程, 但也为微生态研究提供了一种全新的 技术手段。

菌群在肠道中的动态定殖过程一直是研究的 难点, 在人体或普通实验动物上是难以实现的。研 究者们将荧光标记的细菌菌株与无菌斑马鱼幼体 一起培养, 然后利用苂光显微技术获取标记细菌在 斑马鱼肠道中生长的三维图像, 并利用苂光强度实 时测定细菌的多度。这项研究充分发挥了斑马鱼作 为动物模型的优势, 在活的脊椎动物肠道内测定了 微生物生长的动力学数据, 证实其符合对数生长模 型, 并且在空间分布上并不均匀, 而是依赖于不同 位置的微生物密度。这是首次巧妙地结合无菌斑马 鱼模型和荧光显微技术对微生物在肠道内部的空 间结构及生长动态进行研究(Jemielita et al, 2014)。 随后, 研究者还利用斑马鱼模型开展了多项研究, 验证了不同肠道细菌物种对宿主免疫系统的影响 (Rolig et al, 2015), 肠道细菌在宿主其他器官发育 过程中所起的重要作用(Burns et al, 2016; Stephens et al, 2016)。更为重要的是, 越来越多的科研转化实 验也开始在斑马鱼实验平台上开展, 研究者们利用 这一动物模型证实了肠道菌群对于宿主肠道动力 的影响(Wiles et al, 2016), 益生菌对于肥胖和糖尿 病症状的改善(Falcinelli et al, 2017), 甚至是对于抑 郁和压力症状也有一定的干预作用(Davis et al, 2016)。可以说, 在宏基因组研究领域, 不论是基础 研究还是应用转化, 斑马鱼模型都发挥了重要作用, 为研究者们提供了便捷、可靠的研究工具。

但值得注意的是, 斑马鱼模型在宏基因组方面 的应用也存在一些缺点和局限。已有的数据表明, 有近 $70 \%$ 的人体基因可在斑马鱼中找到至少一个直 系同源基因(Howe et $\mathrm{al}, 2013$ ), 证实了该种模式动 物与人类在基因组层面的相似性。但对于宏基因组 研究来说, 共生微生物的组成受环境因素的影响要 大得多。首先, 斑马鱼生存于水生环境中, 这会显 著影响其肠道或其他部位定殖的微生物种类, 造成
其与人体共生微生物之间产生了较大的差异; 其次, 饮食会对肠道微生物造成强烈的影响，这一点是研 究者们所公认的。而斑马鱼的食性与人类完全不同, 因此，饲养过程中所使用的饲料成分也会显著影响 其肠道微生物的组成。研究者们还特地对在不同实 验室饲养的斑马鱼肠道微生物进行了比较, 结果发 现不同实验室来源的斑马鱼, 其肠道菌群存在显著 差异(Roeselers et al, 2011)。此外，从实验技术层面 来说, 斑马鱼体型细小, 基本上不可能进行个体操 作，并且在实验取材上也存在困难，对于粪便样本， 往往是通过解剖获得肠道内容物, 这一做法的直接 后果是粪便细菌DNA中混杂了大量其他来源的核 酸物质, 对后续的测序及数据分析造成较大影响。

\section{2 小鼠(Mus musculus)模型}

不论是在健康还是疾病状况下, 要探索人体自 身的生物学问题, 都需要特定的观察对象和大量的 实验素材。但不论科学家们如何努力, 这一领域研 究总是面临着诸多固有的困难: 人为引入致病或毒 性物质，在伦理上是严格禁止的; 很难从人体获得 所需要的各种组织样本; 人体的某些特定时期, 例 如胚胎期, 是极难被研究者所接触到的。除此之外, 从遗传学角度来看, 人类的代际相对较长, 很难开 展长期的遗传学研究; 此外, 几乎不可能控制某一 人群的配偶关系, 使其满足遗传学研究对其后代遗 传背景的要求。尽管体外细胞培养系统可以解决部 分问题, 但仍然难以再现有机体的整体性及生物体 各系统之间的相互作用(Paigen, 2003)。因此，研究 者们需要一种合适的动物来替代人体作为研究 对象。

代际时间短、繁殖快速、可形成近交系、饲养 方便, 使得小鼠成为较为理想的哺乳动物研究对 象。另外, 小鼠还有一个不容忽视的优点: 成本低 廉。早在1981年，第一只转基因小鼠在实验室的诞 生, 新型的分子生物学技术与传统的小鼠遗传学系 统相结合, 标志着哺乳动物生物学在分子水平的研 究多了一项有力的工具。而小鼠模型与人体的各项 相关性研究也已具备了较为完备的技术基础。发展 至今, 数百种小鼠近交品系、多种突变型、众多的遗 传图谱(Paigen, 2003), 已经构成了一个复杂的遗传 体系, 为各领域的研究者提供了可靠的科研材料。

在漫长的进化过程中, 作为人类祖先的灵长类 动物和作为小鼠祖先的啮齿类动物基因被证实早 
在9,000万年前就已经“分道扬镀” (Lucas et al, 2018), 但二者的基因组仍有 $85 \%$ 以上的相似度(Church et al, 2009)。而转录组分析也发现, 人和小鼠在转录因 子及调控网络上也存在高度的保守性(Cheng et al, 2014)。比较而言, 在人体功能研究当中, 小鼠模型 是更优于体外细胞实验的一种研究手段。肠道细菌 在食物消化过程中发挥重要作用, 因此它们与宿主 营养状况密切相关。营养学领域的研究者们很早就 开始关注肠道菌群在宿主营养方面的作用, 早在20 世纪三、四十年代, 已经有相关的研究探索磺胺类 药物(Najjar, 1943; Hosp, 1945), 抗生素(Emerson et al, 1945; Smith et al, 1945), 以及特定饮食(Friedman, 1936)和肠道细菌之间的关系, 这些研究均在鼠类 模型上开展。如前所述, 20 世纪80年代小鼠遗传学 的快速发展, 产生了各种近交系及人工突变品种, 也为小鼠模型的广泛应用奠定了基础。尽管测序技 术还十分滞后, 当时的研究者们也开始利用16S技 术探索小鼠肠道细菌的组成, 并对其中的特定物种 进行分离、研究(Snel et al, 1995), 而基于小鼠和人 在基因组及转录、调控方面的相似性, 宿主和肠道 菌群之间的相互作用也成为了研究者们的关注重 点。通过无菌鼠和普通小鼠的对比, 研究者们发现 小肠表皮细胞糖基化功能的实现, 需要有肠道细菌 的存在(Bry et al, 1996), 肠道细菌和宿主本身之间 存在着共同进化的关系，二者相互影响，为特定生 理功能研究和新的治疗方法的开发提供了潜在可 能(Hooper \& Gordon, 2001)。而无菌小鼠(germ free mice)模型也在肠道微生物研究中发挥了重要作用, 为解析肠道菌群对宿主健康特别是代谢方面的影 响提供了有力的证据(Rabot et al, 2010; Grover \& Kashyap, 2014; Baothman et al, 2016; Kübeck et al, 2016)。

尽管小鼠模型在各领域科学研究中发挥着无 可替代的作用, 但随着研究的广泛开展, 人们也越 来越深入地了解到小鼠本身的一些局限性。如前所 述, 人和小鼠在自身转录因子及调控网络上存在高 度的保守性。但也有研究揭示, 在小鼠基因组中, 有将近一半的转录因子结合位点未在人类基因组 中找到同源序列，并且整体的基因表达情况在这两 个物种间也存在较大差异(Cheng et al, 2014)。而在 肠道微生物研究领域, 类似的问题也同样存在。在 消化道的生理结构上, 小鼠和人体就存在一些差异,
其中最大的不同就是在小鼠的消化道中存在一个 前胃(forestomach), 其角质化的鳞片粘膜区别于正 常胃部组织, 并且覆盖了整个胃部的 $2 / 3$, 其作用主 要是食物的储存(Ghoshal \& Bal, 1989)。这样的结构 差异导致了其中微生物物种的不同, 有研究发现, 存在于小鼠消化道中的罗伊氏乳杆菌(Lactobacillus reuteri)和在人消化道中发现的同种细菌在基因组 上存在着较大差异(Frese et al, 2011)。

随着高通量测序技术的发展, 研究者们得以对 小鼠和人的肠道细菌组成进行深入的研究和比较。 在门水平上, 二者呈现出较高的相似度, 拟杆菌门 和厚壁菌门均为优势物种(Ley et al, 2006; Rawls et al, 2006)。但需要注意的是, 脱铁杆菌门在小鼠肠道 中定殖较多，而在人肠道中极为少见，只在胃部少 量存在(Bik et al, 2006)。此外，还有一类值得关注的 物种: 分枝丝状杆菌(segmented filamentous bacteria, SFB)。此细菌被多次报道与固有免疫系统(innate immune system)的成熟密切相关(Suzuki et al, 2004; Gaboriau-Routhiau et al, 2009; Ivanov et al, 2009), 此物种在小鼠肠道中较为常见, 但几乎没有在成人 的肠道中发现。

而针对大规模小鼠肠道样本的宏基因组研究 也对小鼠肠道细菌的组成进行了深入研究(Xiao et al, 2015)，并与已报道的人肠道细菌分布(Qin et al, 2010)进行了比较, 在总共60个核心细菌属中, 在小 鼠和人肠道中同时存在的仅有 25 个, 且丰度各有不 同。在微生物基因方面，二者差异更为巨大，仅有 4\%的微生物基因同时存在于二者的微生物基因集 合当中。但值得注意的是，尽管小鼠和人类的肠道 菌群在物种和基因上差异甚大，但在功能层面却具 有较高的相似性，二者共享了超过 $80 \%$ 的可注释功 能。目前为止, 已有超过 1,500 株分离自人肠道的细 菌菌株被用于深入研究(Rajilic-Stojanovic \& de Vos, 2014; Lagier et al, 2016), 而只有100株左右来自小 鼠肠道的细菌被分离出来(Lagkouvardos et al, 2016)。对于小鼠肠道微生物的研究，还有很多工作 要做。

小鼠作为研究工具的优势冊庸置疑，这也是它 成为最广泛使用的实验模型的原因。与此同时，当 我们将小鼠应用于肠道宏基因组领域时，也有一些 值得注意的限制。如前所述，小鼠和人肠道本身在 肠道微生物组成方面存在先天的差异，在将小鼠实 
验的结果向人体外推时, 这些差异是需要关注的。 无菌小鼠的出现, 部分缩小了小鼠和人之间在肠道 微生态方面的差异, 研究者们将来源于人的肠道菌 群移植进无菌小鼠的肠道中, 发现有 $88 \%$ 的人源细 菌属可以在无菌小鼠的肠道中定殖(Turnbaugh et al, 2009)。而这一技术最著名的应用在于以肥胖为代表 的代谢性疾病研究(Ley et al, 2005; Geurts et al, 2011; Wang et al, 2017), 证实了肠道菌群与代谢性疾病之 间的因果关系。但需要注意的是, 在高度流程化的 小鼠实验过程中, 饲喂过程对于小鼠肠道微生态的 影响极为巨大, 进而可能影响到最终的实验结果。 在某些情况下, 鼠房的更新和改造, 会导致原本可 观察的某些表型完全消失, 饲喂环境的影响甚至比 遗传背景还要大(Friswell et al, 2010; Verbeke et al, 2015; Xiao et al, 2015)。这不得不引起人们的注意, 也是研究者在应用小鼠模型时所必须考虑的问题。

大鼠作为小鼠的“近亲”, 因为操作复杂度和成 本等原因, 在微生态研究领域的应用广泛程度不及 小鼠, 但因为二者的相似程度, 其特点和优缺点比 较类似。也有研究者对SD (Sprague-Dawley)大鼠的 肠道微生物特征进行了研究, 将人、小鼠、大鼠的 肠道微生物数据集合进行了比较, 发现大鼠与人之 间共享的肠道微生物基因数量和功能基因簇数量 要略高于小鼠(Pan et al, 2018)。

\section{3 猪(Sus scrofa domesticus)模型和猕猴(Macaca mulatta)模型}

除了上述的斑马鱼和小鼠, 猪作为一种大型哺 乳动物也在很多科学研究中被作为模型使用。实际 上, 作为人类重要的食物来源之一, 关于猪本身的 各种营养和疾病相关问题早已经成为研究者们关 注的领域。和小鼠类似, 营养学家们最早开始关注 猪肠道菌群和宿主营养之间的联系。早在1936年, 就已经有了关于无菌猪技术的相关报道(Glimstedt, 1936), 随后的研究也将猪和人的肠道微生物进行 了比较, 发现其在基因、物种和功能层面, 确实比 小鼠更接近于人的肠道(Hildebrand et al, 2012; Xiao et al, 2016), 并且在消化系统及食性方面, 猪和人 之间也更为相似。但和小型动物相比, 猪作为动物 模型在研究应用中最大的问题在于维持成本较高。 由于体型过大, 对饲喂场地要求较高; 指标测量、 组织采样等操作也远比小型动物繁琐, 这些因素都 限制了猪模型的使用范围。但随着遗传技术特别是
分子遗传技术的发展, 研究者们已经开始针对猪这 个物种进行各种遗传操作，以符合科研或产业应用 的需求(Sachs \& Galli, 2009), 这其中也包括小型化 (Cyranoski, 2015), 这样的操作还存在一定的问题 和争议, 但体型的缩小无疑解决了猪作为研究对象 的一个关键性问题。

作为和人类最为接近的灵长类动物, 猴子无疑 也是理想的模型动物。在医药研究中, 猩猩、恒河 猴等非人灵长类(nonhuman primates, NHPs)动物为 各类药物、疫苗的开发做出了巨大贡献。猴模型在 肠道宏基因组中的应用比较晚，但近年来也开始被 广泛用于研究饮食、抗生素、遗传背景等对于肠道 微生物的影响(Angelakis et al, 2016; Harris et al, 2016; Zevin et al, 2017)。通过人肠道微生态的比较, 也确实证实了白慰叶猴(Pygathrix nemaeus)、鬃毛吼 猴(Alouatta palliata)、食蟹猴(Macaca fascicularis) 在肠道微生物组成上较高的一致性(Clayton et al, 2016; Li et al, 2018)。与猪模型一样，猴模型的应用 也受到饲喂环境和成本的制约，难以像小型动物那 样大规模使用。并且也有研究报道, 笼养的NHPs 肠道微生物与野生状态下存在较大差异(Clayton et al，2016), 这其实也是所有模型动物在应用于宏基 因组研究时所共同面临的问题：同一类模型动物, 不同的饲喂场所，环境因素往往会造成肠道微生态 的差异, 可能造成实验结果的不稳定(Stappenbeck \& Virgin, 2016), 这是在将动物模型应用于宏基因 组研究时需要考虑的因素。而大型动物由于饲养环 境复杂、控制度较低, 这一因素的影响会更为明显。

\section{新兴动物模型及展望}

传统的临床微生物研究主要关注某一种病原 微生物对人类疾病的影响和作用, 随着高通量测序 技术的突破，人们已经可以针对特定环境中的微生 物群落进行研究。现在已经了解到, 人体共生微生 物由多种多样的细菌、古菌及真菌组成，它们中的 绝大多数是非病原菌, 但却通过各种机制、途径影 响着宿主的健康，包括：营养及药物代谢(Wallace et al, 2010; de Clercq et al, 2016)、必需维生素的合成 (Kau et al, 2011)、抵抗病原菌(Abt \& Pamer, 2014)、 宿主胆汁酸的次级代谢(Wahlström et al, 2016)、免 疫调控(Round \& Mazmanian, 2009; Belkaid \& Hand, 2014)、感染的抵抗或敏感(Buffie et al, 2015), 甚至 
宿主行为的调控(Dinan et al, 2015)。因为与人体健 康之间的密切联系, 使得以共生微生物为主要研究 对象的宏基因组学成为了近年来的研究焦点。

在宏基因组学研究过程中, 动物模型作为不可 或缺的研究工具, 自然也受到人们的重视。如前所 述, 研究者们不断尝试新技术、新方法, 将动物模 型应用到多种共生微生物(主要为肠道微生物)的研 究中, 并取得了众多的研究成果, 其中一些结论 甚至颠覆了人们之前的普遍认识。毫无疑问, 模型 动物为这一领域做出了重要贡献。但值得注意的是, 模型动物的使用过程中也存在一些固有的问题和 困难, 以至于它们本身也成为了研究目的。研究者 们同样进行了大量研究来探索如何更经济、更准确 地使用模型动物。本文综述了一些常见的模型动物 在宏基因组领域的研究情况, 以及值得注意的问题 和优缺点。实际上, 模型动物的范围还在不断扩展, 不断有新兴的模型被应用于特定领域的研究作为 农业甚至整个生态环境的重要一员, 蜜蜂(Apis)的 肠道微生态早已引起了研究者的兴趣(Cox-Foster et $\mathrm{al}, 2007)$, 并且蜜蜂作为一种较为新颖的模型昆虫 也已经被应用在行为及认知功能方面的研究中 (Menzel, 2012)。蜜蜂肠道菌群结构较为简单, 繁殖 和饲养相比大型动物来说更好操控, 这些优点十分 有利于将其应用于共生微生物的研究当中(Engel et al, 2016)。同样地, 果蝇作为十分成熟的模型昆虫, 被广泛应用在遗传学等多个领域的研究中(Adams et al, 2000; Reiter et al, 2001; Valente et al, 2004; Lloyd \& Taylor, 2010), 且研究者们基于该种模式生 物构建了多种疾病模型(Pandey \& Nichols, 2011), 包括二型糖尿病模型(Álvarez-Rendón et al, 2018), 越来越多的基于果蝇的微生态研究(Clark et al, 2015; Leftwich et al, 2018; Obadia et al, 2018)也预示 着其作为该领域新型研究工具的重要性。秀丽隐杆 线虫(Caenorhabditis elegans)作为一种经典的模式 生物, 其生命周期只有数周, 在这期间完成成长、 繁殖、衰老, 最终死亡的整个过程, 研究者们依据 线虫的这一特点, 研究了肠道微生物特定基因对线 虫寿命的影响, 取得了非常显著的进展(Han et al, 2017)。此外, 线虫还被用于研究肠道微生物在癌症 化疗中的作用(García-González et al, 2017; Scott et al, 2017), 展现了其作为经典模式生物在微生态这 一新兴领域所发挥的重要作用。与此同时, 体外技
术也在不断发展, 类器官、器官芯片等新技术的出 现，让研究者们有了更多的选择。可以预见，共生 微生物领域的研究将随着新模型、新技术的出现进 入全新的阶段。

\section{参考文献}

Abt MC, Pamer EG (2014) Commensal bacteria mediated defenses against pathogens. Current Opinion in Immunology, 29, 16-22.

Adams MD, Celniker SE, Holt RA, Evans CA, Gocayne JD, Amanatides PG, Scherer SE, Li PW, Hoskins RA, Galle RF, George RA, Lewis SE, Richards S, Ashburner M, Henderson SN, Sutton GG, Wortman JR, Yandell MD, Zhang Q, Chen LX, Brandon RC, Rogers YH, Blazej RG, Champe M, Pfeiffer BD, Wan KH, Doyle C, Baxter EG, Helt G, Nelson CR, Gabor GL, Abril JF, Agbayani A, An HJ, Andrews-Pfannkoch C, Baldwin D, Ballew RM, Basu A, Baxendale J, Bayraktaroglu L, Beasley EM, Beeson KY, Benos PV, Berman BP, Bhandari D, Bolshakov S, Borkova D, Botchan MR, Bouck J, Brokstein P, Brottier P, Burtis KC, Busam DA, Butler H, Cadieu E, Center A, Chandra I, Cherry JM, Cawley S, Dahlke C, Davenport LB, Davies P, de Pablos B, Delcher A, Deng Z, Mays AD, Dew I, Dietz SM, Dodson K, Doup LE, Downes M, Dugan-Rocha S, Dunkov BC, Dunn P, Durbin KJ, Evangelista CC, Ferraz C, Ferriera S, Fleischmann W, Fosler C, Gabrielian AE, Garg NS, Gelbart WM, Glasser K, Glodek A, Gong F, Gorrell JH, Gu Z, Guan P, Harris M, Harris NL, Harvey D, Heiman TJ, Hernandez JR, Houck J, Hostin D, Houston KA, Howland TJ, Wei MH, Ibegwam C, Jalali M, Kalush F, Karpen GH, Ke Z, Kennison JA, Ketchum KA, Kimmel BE, Kodira CD, Kraft C, Kravitz S, Kulp D, Lai Z, Lasko P, Lei Y, Levitsky AA, Li J, Li Z, Liang Y, Lin X, Liu X, Mattei B, McIntosh TC, McLeod MP, McPherson D, Merkulov G, Milshina NV, Mobarry C, Morris J, Moshrefi A, Mount SM, Moy M, Murphy B, Murphy L, Muzny DM, Nelson DL, Nelson DR, Nelson KA, Nixon K, Nusskern DR, Pacleb JM, Palazzolo M, Pittman GS, Pan S, Pollard J, Puri V, Reese MG, Reinert K, Remington K, Saunders RD, Scheeler F, Shen H, Shue BC, Sidén-Kiamos I, Simpson M, Skupski MP, Smith T, Spier E, Spradling AC, Stapleton M, Strong R, Sun E, Svirskas R, Tector C, Turner R, Venter E, Wang AH, Wang $\mathrm{X}$, Wang ZY, Wassarman DA, Weinstock GM, Weissenbach J, Williams SM, Woodage T, Worley KC, Wu D, Yang S, Yao QA, Ye J, Yeh RF, Zaveri JS, Zhan M, Zhang G, Zhao Q, Zheng L, Zheng XH, Zhong FN, Zhong W, Zhou X, Zhu S, Zhu X, Smith HO, Gibbs RA, Myers EW, Rubin GM, Venter JC (2000) The genome sequence of Drosophila melanogaster. Science, 287, 2185-2195.

Al-Shehri SS, Sweeney EL, Cowley DM, Liley HG, Ranasinghe PD, Charles BG, Shaw PN, Vagenas D, Duley JA, 
Knox CL (2016) Deep sequencing of the $16 \mathrm{~S}$ ribosomal RNA of the neonatal oral microbiome: A comparison of breast-fed and formula-fed infants. Scientific Reports, 6, 38309.

Álvarez-Rendón JP, Salceda R, Riesgo-Escovar JR (2018) Drosophila melanogaster as a model for diabetes type 2 progression. BioMed Research International, 8, 1417528.

Angelakis E, Yasir M, Bachar D, Azhar EI, Lagier JC, Bibi F, Jiman-Fatani AA, Alawi M, Bakarman MA, Robert C, Raoult D (2016) Gut microbiome and dietary patterns in different Saudi populations and monkeys. Scientific Reports, 6, 32191.

Baothman OA, Zamzami MA, Taher I, Abubaker J, Abu-Farha M (2016) The role of gut microbiota in the development of obesity and diabetes. Lipids in Health and Disease, 15, 108-116.

Belkaid Y, Hand TW (2014) Role of the microbiota in immunity and inflammation. Cell, 157, 121-141.

Berer K, Gerdes LA, Cekanaviciute E, Jia X, Xiao L, Xia Z, Liu C, Klotz L, Stauffer U, Baranzini SE, Kümpfel T, Hohlfeld R, Krishnamoorthy G, Wekerle H (2017) Gut microbiota from multiple sclerosis patients enables spontaneous autoimmune encephalomyelitis in mice. Proceedings of the National Academy of Sciences, USA, 114, 10719-10724.

Bik EM, Eckburg PB, Gill SR, Nelson KE, Purdom EA, Francois F, Perez-Perez G, Blaser MJ, Relman DA (2006) Molecular analysis of the bacterial microbiota in the human stomach. Proceedings of the National Academy of Sciences, USA, 103, 732-737.

Bry L, Falk PG, Midtvedt T, Gordon JI (1996) A model of host-microbial interactions in an open mammalian ecosystem. Science, 273, 1380-1383.

Buffie CG, Bucci V, Stein RR, McKenney PT, Ling L, Gobourne A, No D, Liu H, Kinnebrew M, Viale A, Littmann E, van den Brink MRM, Jenq RR, Taur Y, Sander C, Cross JR, Toussaint NC, Xavier JB, Pamer EG (2015) Precision microbiome reconstitution restores bile acid mediated resistance to Clostridium difficile. Nature, 517, 205-208.

Burns AR, Stephens WZ, Stagaman K, Wong S, Rawls JF, Guillemin K, Bohannan BJ (2016) Contribution of neutral processes to the assembly of gut microbial communities in the zebrafish over host development. The ISME Journal, 10, 655-664.

Cheng Y, Ma Z, Kim B-H, Wu W, Cayting P, Boyle AP, Sundaram V, Xing X, Dogan N, Li J, Euskirchen G, Lin S, Lin Y, Visel A, Kawli T, Yang X, Patacsil D, Keller CA, Giardine B, Consortium TME, Kundaje A, Wang T, Pennacchio LA, Weng Z, Hardison RC, Snyder MP (2014) Principles of regulatory information conservation between mouse and human. Nature, 515, 371-375.

Church DM, Goodstadt L, Hillier LW, Zody MC, Goldstein S, She X, Bult CJ, Agarwala R, Cherry JL, DiCuccio M,
Hlavina W, Kapustin Y, Meric P, Maglott D, Birtle Z, Marques AC, Graves T, Zhou S, Teague B, Potamousis K, Churas C, Place M, Herschleb J, Runnheim R, Forrest D, Amos-Landgraf J, Schwartz DC, Cheng Z, Lindblad-Toh K, Eichler EE, Ponting CP, Consortium TMGS (2009) Lineage-specific biology revealed by a finished genome assembly of the mouse. PLoS Biology, 7, e1000112.

Clark RI, Salazar A, Yamada R, Fitz-Gibbon S, Morselli M, Alcaraz J, Rana A, Rera M, Pellegrini M, Ja WW, Walker DW (2015) Distinct shifts in microbiota composition during Drosophila aging impair intestinal function and drive mortality. Cell Reports, 12, 1656-1667.

Clayton JB, Vangay P, Huang H, Ward T, Hillmann BM, Al-Ghalith GA, Travis DA, Long HT, Tuan BV, Minh VV, Cabana F, Nadler T, Toddes B, Murphy T, Glander KE, Johnson TJ, Knights D (2016) Captivity humanizes the primate microbiome. Proceedings of the National Academy of Sciences, USA, 113, 10376-10381.

Clemente JC, Ursell LK, Parfrey LW, Knight R (2012) The impact of the gut microbiota on human health: An integrative view. Cell, 148, 1258-1270.

Cox-Foster DL, Conlan S, Holmes EC, Palacios G, Evans JD, Moran NA, Quan PL, Briese T, Hornig M, Geiser DM, Martinson V, vanEngelsdorp D, Kalkstein AL, Drysdale A, Hui J, Zhai J, Cui L, Hutchison SK, Simons JF, Egholm M, Pettis JS, Lipkin WI (2007) A metagenomic survey of microbes in honey bee colony collapse disorder. Science, 318, 283-287.

Cyranoski D (2015) Gene-edited “micropigs” to be sold as pets at Chinese institute. Nature News, 526, 18.

Davis DJ, Doerr HM, Grzelak AK, Busi SB, Jasarevic E, Ericsson AC, Bryda EC (2016) Lactobacillus plantarum attenuates anxiety-related behavior and protects against stress-induced dysbiosis in adult zebrafish. Scientific Reports, 6, 33726.

de Clercq NC, Groen AK, Romijn JA, Nieuwdorp M (2016) Gut microbiota in obesity and undernutrition. Advances in Nutrition, 7, 1080-1089.

Dinan TG, Stilling RM, Stanton C, Cryan JF (2015) Collective unconscious: How gut microbes shape human behavior. Journal of Psychiatric Research, 63, 1-9.

Emerson GA, Smith DG, Wurtz E, Clark D (1945) Induction of nutritional deficiency by oral administration of streptomycin. Journal of Pharmacology and Experimental Therapeutics, 85, 336-342.

Engel P, Kwong WK, McFrederick Q, Anderson KE, Barribeau SM, Chandler JA, Cornman RS, Dainat J, de Miranda JR, Doublet V, Emery O, Evans JD, Farinelli L, Flenniken ML, Granberg F, Grasis JA, Gauthier L, Hayer J, Koch H, Kocher S, Martinson VG, Moran N, Munoz-Torres M, Newton I, Paxton RJ, Powell E, Sadd BM, Schmid-Hempel P, Schmid-Hempel R, Song SJ, Schwarz RS, vanEngelsdorp D, Dainat B (2016) The bee microbiome: Impact on bee 
health and model for evolution and ecology of host-microbe interactions. mBio, 7, e02164-15.

Falcinelli S, Rodiles A, Hatef A, Picchietti S, Cossignani L, Merrifield DL, Unniappan S, Carnevali O (2017) Dietary lipid content reorganizes gut microbiota and probiotic $L$. rhamnosus attenuates obesity and enhances catabolic hormonal milieu in zebrafish. Scientific Reports, 7, 5512.

Frese SA, Benson AK, Tannock GW, Loach DM, Kim J, Zhang M, Oh PL, Heng NCK, Patil PB, Juge N, MacKenzie DA, Pearson BM, Lapidus A, Dalin E, Tice H, Goltsman E, Land M, Hauser L, Ivanova N, Kyrpides NC, Walter J (2011) The evolution of host specialization in the vertebrate gut symbiont Lactobacillus reuteri. PLoS Genetics, 7, e1001314.

Friedman H (1936) A study of the fecal flora and the line test of normal rats, rachitic rats and heating rachitic rats. Journal of Nutrition, 12, 165-172.

Friswell MK, Gika H, Stratford IJ, Theodoridis G, Telfer B, Wilson ID, McBain AJ (2010) Site and strain-specific variation in gut microbiota profiles and metabolism in experimental mice. PLoS ONE, 5, e8584.

Gaboriau-Routhiau V, Rakotobe S, Lécuyer E, Mulder I, Lan A, Bridonneau C, Rochet V, Pisi A, De Paepe M, Brandi G, Eberl G, Snel J, Kelly D, Cerf-Bensussan N (2009) The key role of segmented eilamentous bacteria in the coordinated maturation of gut helper $\mathrm{T}$ cell responses. Immunity, 31, 677-689.

García-González AP, Ritter AD, Shrestha S, Andersen EC, Yilmaz LS, Walhout AJM (2017) Bacterial metabolism affects the $C$. elegans response to cancer chemotherapeutics. Cell, 169, 431-441.

Geurts L, Lazarevic V, Derrien M, Everard A, Van Roye M, Knauf C, Valet P, Girard M, Muccioli GG, Francois P, de Vos WM, Schrenzel J, Delzenne NM, Cani PD (2011) Altered gut microbiota and endocannabinoid system tone in obese and diabetic leptin-resistant mice: Impact on apelin regulation in adipose tissue. Frontiers in Microbiology, 2, https://doi.org/10.3389/fmicb.2011.00149.

Ghoshal NG, Bal HS (1989) Comparative morphology of the stomach of some laboratory mammals. Laboratory Animals, 23, 21-29.

Glimstedt G (1936) Der Stoffwechsel bakterienfreier Tiere1. Skandinavisches Archiv Für Physiologie, 73, 48-62. (in German)

Grover M, Kashyap PC (2014) Germ-free mice as a model to study effect of gut microbiota on host physiology. Neurogastroenterology \& Motility, 26, 745-748.

Han B, Sivaramakrishnan P, Lin CJ, Neve IAA, He J, Tay LWR, Sowa JN, Sizovs A, Du G, Wang J, Herman C, Wang MC (2017) Microbial genetic composition tunes host longevity. Cell, 169, 1249-1262.

Haque MM, Merchant M, Kumar PN, Dutta A, Mande SS (2017) First-trimester vaginal microbiome diversity: A potential indicator of preterm delivery risk. Scientific Reports, 7, 16145.

Harris RA, Alcott CE, Sullivan EL, Takahashi D, McCurdy CE, Comstock S, Baquero K, Blundell P, Frias AE, Kahr M, Suter M, Wesolowski S, Friedman JE, Grove KL, Aagaard KM (2016) Genomic variants associated with resistance to high fat diet induced obesity in a primate model. Scientific Reports, 6, 36123.

Hildebrand F, Ebersbach T, Nielsen HB, Li X, Sonne SB, Bertalan M, Dimitrov P, Madsen L, Qin J, Wang J, Raes J, Kristiansen K, Licht TR (2012) A comparative analysis of the intestinal metagenomes present in Guinea pigs (Cavia porcellus) and humans (Homo sapiens). BMC Genomics, 13, 514.

Hooper LV, Gordon JI (2001) Commensal host-bacterial relationships in the gut. Science, 292, 1115-1118.

Hosp H (1945) Sulfonamides and vitamin K deficiency. Nutrition Reviews, 3, 35-36.

Howe K, Clark MD, Torroja CF, Torrance J, Berthelot C, Muffato M, Collins JE, Humphray S, McLaren K, Matthews L, McLaren S, Sealy I, Caccamo M, Churcher C, Scott C, Barrett JC, Koch R, Rauch GJ, White S, Chow W, Kilian B, Quintais LT, Guerra-Assunção JA, Zhou Y, Gu Y, Yen J, Vogel JH, Eyre T, Redmond S, Banerjee R, Chi J, Fu B, Langley E, Maguire SF, Laird GK, Lloyd D, Kenyon E, Donaldson S, Sehra H, Almeida-King J, Loveland J, Trevanion S, Jones M, Quail M, Willey D, Hunt A, Burton J, Sims S, McLay K, Plumb B, Davis J, Clee C, Oliver K, Clark R, Riddle C, Elliot D, Threadgold G, Harden G, Ware D, Begum S, Mortimore B, Kerry G, Heath P, Phillimore B, Tracey A, Corby N, Dunn M, Johnson C, Wood J, Clark S, Pelan S, Griffiths G, Smith M, Glithero R, Howden P, Barker N, Lloyd C, Stevens C, Harley J, Holt K, Panagiotidis G, Lovell J, Beasley H, Henderson C, Gordon D, Auger K, Wright D, Collins J, Raisen C, Dyer L, Leung K, Robertson L, Ambridge K, Leongamornlert D, McGuire S, Gilderthorp R, Griffiths C, Manthravadi D, Nichol S, Barker G, Whitehead S, Kay M, Brown J, Murnane C, Gray E, Humphries M, Sycamore N, Barker D, Saunders D, Wallis J, Babbage A, Hammond S, Mashreghi-Mohammadi M, Barr L, Martin S, Wray P, Ellington A, Matthews N, Ellwood M, Woodmansey R, Clark G, Cooper J, Tromans A, Grafham D, Skuce C, Pandian R, Andrews R, Harrison E, Kimberley A, Garnett J, Fosker N, Hall R, Garner P, Kelly D, Bird C, Palmer S, Gehring I, Berger A, Dooley CM, Ersan-Ürün Z, Eser C, Geiger H, Geisler M, Karotki L, Kirn A, Konantz J, Konantz M, Oberländer M, Rudolph-Geiger S, Teucke M, Lanz C, Raddatz G, Osoegawa K, Zhu B, Rapp A, Widaa S, Langford C, Yang F, Schuster SC, Carter NP, Harrow J, Ning Z, Herrero J, Searle SM, Enright A, Geisler R, Plasterk RH, Lee C, Westerfield M, de Jong PJ, Zon LI, Postlethwait JH, Nüsslein-Volhard C, Hubbard TJ, Roest Crollius H, Rogers J, Stemple DL (2013) The 
zebrafish reference genome sequence and its relationship to the human genome. Nature, 496, 498-503.

Ivanov II, Atarashi K, Manel N, Brodie EL, Shima T, Karaoz U, Wei D, Goldfarb KC, Santee CA, Lynch SV, Tanoue T, Imaoka A, Itoh K, Takeda K, Umesaki Y, Honda K, Littman DR (2009) Induction of intestinal Th17 cells by segmented filamentous bacteria. Cell, 139, 485-498.

Jemielita M, Taormina MJ, Burns AR, Hampton JS, Rolig AS, Guillemin K, Parthasarathy R (2014) Spatial and temporal features of the growth of a bacterial species colonizing the zebrafish gut. mBio, 5, e01751-14.

Jie Z, Xia H, Zhong S-L, Feng Q, Li S, Liang S, Zhong H, Liu Z, Gao Y, Zhao H, Zhang D, Su Z, Fang Z, Lan Z, Li J, Xiao L, Li J, Li R, Li X, Li F, Ren H, Huang Y, Peng Y, Li G, Wen B, Dong B, Chen JY, Geng QS, Zhang ZW, Yang H, Wang J, Wang J, Zhang X, Madsen L, Brix S, Ning G, Xu X, Liu X, Hou Y, Jia H, He K, Kristiansen K (2017) The gut microbiome in atherosclerotic cardiovascular disease. Nature Communications, 8, 845.

Kau AL, Ahern PP, Griffin NW, Goodman AL, Gordon JI (2011) Human nutrition, the gut microbiome, and immune system: Envisioning the future. Nature, 474, 327-336.

Keller PJ, Schmidt AD, Wittbrodt J, Stelzer EHK (2008) Reconstruction of zebrafish early embryonic development by scanned light sheet microscopy. Science, 322, 1065-1069.

Kelly S (2016) Mouse microbes may make scientific studies harder to replicate. Science, 353, 741-743.

Kilian M, Chapple ILC, Hannig M, Marsh PD, Meuric V, Pedersen AML, Tonetti MS, Wade WG, Zaura E (2016) The oral microbiome-An update for oral healthcare professionals. British Dental Journal, 221, 657-666.

Kübeck R, Bonet-Ripoll C, Hoffmann C, Walker A, Müller VM, Schüppel VL, Lagkouvardos I, Scholz B, Engel K-H, Daniel H, Schmitt-Kopplin P, Haller D, Clavel T, Klingenspor M (2016) Dietary fat and gut microbiota interactions determine diet-induced obesity in mice. Molecular Metabolism, 5, 1162-1174.

Lagier J-C, Khelaifia S, Alou MT, Ndongo S, Dione N, Hugon P, Caputo A, Cadoret F, Traore SI, Seck EH, Dubourg G, Durand G, Mourembou G, Guilhot E, Togo A, Bellali S, Bachar D, Cassir N, Bittar F, Delerce J, Mailhe M, Ricaboni D, Bilen M, Dangui Nieko NPM, Dia Badiane NM, Valles C, Mouelhi D, Diop K, Million M, Musso D, Abrahão J, Azhar EI, Bibi F, Yasir M, Diallo A, Sokhna C, Djossou F, Vitton V, Robert C, Rolain JM, La Scola B, Fournier P-E, Levasseur A, Raoult D (2016) Culture of previously uncultured members of the human gut microbiota by culturomics. Nature Microbiology, 1, 16203.

Lagkouvardos I, Pukall R, Abt B, Foesel BU, Meier-Kolthoff JP, Kumar N, Bresciani A, Martínez I, Just S, Ziegler C, Brugiroux S, Garzetti D, Wenning M, Bui TPN, Wang J, Hugenholtz F, Plugge CM, Peterson DA, Hornef MW, Baines JF, Smidt H, Walter J, Kristiansen K, Nielsen HB,
Haller D, Overmann J, Stecher B, Clavel T (2016) The Mouse Intestinal Bacterial Collection (miBC) provides host-specific insight into cultured diversity and functional potential of the gut microbiota. Nature Microbiology, 1, 16131.

Leftwich PT, Clarke NVE, Hutchings MI, Chapman T (2018) Reply to Rosenberg et al: Diet, gut bacteria, and assortative mating in Drosophila melanogaster. Proceedings of the National Academy of Sciences, USA, 115, E2154-E2155.

Ley RE, Bäckhed F, Turnbaugh P, Lozupone CA, Knight RD, Gordon JI (2005) Obesity alters gut microbial ecology. Proceedings of the National Academy of Sciences, USA, 102, 11070-11075.

Ley RE, Turnbaugh PJ, Klein S, Gordon JI (2006) Microbial ecology: Human gut microbes associated with obesity. Nature, 444, 1022-1023.

Li X, Liang S, Xia Z, Qu J, Liu H, Liu C, Yang H, Wang J, Madsen L, Hou Y, Li J, Jia H, Kristiansen K, Xiao L (2018) Establishment of a Macaca fascicularis gut microbiome gene catalog and comparison with the human, pig, and mouse gut microbiomes. GigaScience, 7, 9.

Liu R, Hong J, Xu X, Feng Q, Zhang D, Gu Y, Shi J, Zhao S, Liu W, Wang X, Xia H, Liu Z, Cui B, Liang P, Xi L, Jin J, Ying $X$, Wang $X$, Zhao X, Li W, Jia H, Lan Z, Li F, Wang R, Sun Y, Yang M, Shen Y, Jie Z, Li J, Chen X, Zhong H, Xie H, Zhang Y, Gu W, Deng X, Shen B, Xu X, Yang H, Xu G, Bi Y, Lai S, Wang J, Qi L, Madsen L, Wang J, Ning G, Kristiansen K, Wang W (2017) Gut microbiome and serum metabolome alterations in obesity and after weight-loss intervention. Nature Medicine, 23, 859-868.

Lloyd TE, Taylor JP (2010) Flightless flies: Drosophila models of neuromuscular disease. Annals of the New York Academy of Sciences, 1184, E1-E20.

Lucas BA, Lavi E, Shiue L, Cho H, Katzman S, Miyoshi K, Siomi MC, Carmel L, Ares M, Maquat LE (2018) Evidence for convergent evolution of SINE-directed Staufen-mediated mRNA decay. Proceedings of the National Academy of Sciences, USA, 115, 965-973.

Marchesi JR, Adams DH, Fava F, Hermes GDA, Hirschfield GM, Hold G, Quraishi MN, Kinross J, Smidt H, Tuohy KM, Thomas LV, Zoetendal EG, Hart A (2016) The gut microbiota and host health: A new clinical frontier. Gut, 65, 330-339.

Menzel R (2012) The honeybee as a model for understanding the basis of cognition. Nature Reviews Neuroscience, 13, 758-768.

Najjar VA (1943) Vitamin synthesis by intestinal bacteria. Nutrition Reviews, 1, 175-176.

Obadia B, Keebaugh ES, Yamada R, Ludington WB, Ja WW (2018) Diet influences host-microbiota associations in Drosophila. Proceedings of the National Academy of Sciences, USA, 115, E4547-E4548.

Paigen K (2003) One hundred years of mouse genetics: An 
intellectual history. II. The molecular revolution (1981-2002). Genetics, 163, 1227-1235.

Pan H, Guo R, Zhu J, Wang Q, Ju Y, Xie Y, Zheng Y, Wang Z, Li T, Liu Z, Lu L, Li F, Tong B, Xiao L, Xu X, Li R, Yuan Z, Yang H, Wang J, Kristiansen K, Jia H, Liu L (2018) A gene catalogue of the Sprague-Dawley rat gut metagenome. GigaScience, 7, 5.

Pandey UB, Nichols CD (2011) Human disease models in Drosophila melanogaster and the role of the fly in therapeutic drug discovery. Pharmacological Reviews, 63, 411-436.

Qin J, Li R, Raes J, Arumugam M, Burgdorf KS, Manichanh C, Nielsen T, Pons N, Levenez F, Yamada T, Mende DR, Li J, Xu J, Li S, Li D, Cao J, Wang B, Liang H, Zheng H, Xie Y, Tap J, Lepage P, Bertalan M, Batto JM, Hansen T, Le Paslier D, Linneberg A, Nielsen HB, Pelletier E, Renault P, Sicheritz-Ponten T, Turner K, Zhu H, Yu C, Li S, Jian M, Zhou Y, Li Y, Zhang X, Li S, Qin N, Yang H, Wang J, Brunak S, Doré J, Guarner F, Kristiansen K, Pedersen O, Parkhill J, Weissenbach J, MetaHIT Consortium, Bork P, Ehrlich SD, Wang J (2010) A human gut microbial gene catalogue established by metagenomic sequencing. Nature, 464, 59-65.

Qin J, Li Y, Cai Z, Li S, Zhu J, Zhang F, Liang S, Zhang W, Guan Y, Shen D, Peng Y, Zhang D, Jie Z, Wu W, Qin Y, Xue W, Li J, Han L, Lu D, Wu P, Dai Y, Sun X, Li Z, Tang A, Zhong S, Li X, Chen W, Xu R, Wang M, Feng Q, Gong M, Yu J, Zhang Y, Zhang M, Hansen T, Sanchez G, Raes J, Falony G, Okuda S, Almeida M, LeChatelier E, Renault P, Pons N, Batto JM, Zhang Z, Chen H, Yang R, Zheng W, Li S, Yang H, Wang J, Ehrlich SD, Nielsen R, Pedersen O, Kristiansen K, Wang J (2012) A metagenome-wide association study of gut microbiota in Type 2 diabetes. Nature, 490, 55-60.

Rabot S, Membrez M, Bruneau A, Gérard P, Harach T, Moser M, Raymond F, Mansourian R, Chou CJ (2010) Germ-free C57BL/6J mice are resistant to high-fat-diet-induced insulin resistance and have altered cholesterol metabolism. The FASEB Journal, 24, 4948-4959.

Rajilic-Stojanovic M, de Vos WM (2014) The first 1000 cultured species of the human gastrointestinal microbiota. FEMS Microbiology Reviews, 35, 996-1047.

Ramakrishna BS (2013) Role of the gut microbiota in human nutrition and metabolism. Journal of Gastroenterology and Hepatology, 28(Suppl. 4), 9-17.

Rawls JF, Mahowald MA, Ley RE, Gordon JI (2006) Reciprocal gut microbiota transplants from zebrafish and mice to germ-free recipients reveal host habitat selection. Cell, 127, 423-433.

Rawls JF, Samuel BS, Gordon JI (2004) Gnotobiotic zebrafish reveal evolutionarily conserved responses to the gut microbiota. Proceedings of the National Academy of Sciences, USA, 101, 4596-4601.

Reiter LT, Potocki L, Chien S, Gribskov M, Bier E (2001) A systematic analysis of human disease-associated gene sequences in Drosophila melanogaster. Genome Research, 11, 1114-1125.

Roeselers G, Mittge EK, Stephens WZ, Parichy DM, Cavanaugh CM, Guillemin K, Rawls JF (2011) Evidence for a core gut microbiota in the zebrafish. The ISME Journal, 5, 1595-1608.

Rolig AS, Parthasarathy R, Burns AR, Bohannan BJM, Guillemin K (2015) Individual members of the microbiota disproportionately modulate host innate immune responses. Cell Host \& Microbe, 18, 613-620.

Round JL, Mazmanian SK (2009) The gut microbiota shapes intestinal immune responses during health and disease. Nature Reviews Immunology, 9, 313-323.

Rowland I, Gibson G, Heinken A, Scott K, Swann J, Thiele I, Tuohy K (2018) Gut microbiota functions: Metabolism of nutrients and other food components. European Journal of Nutrition, 57, 1-24.

Sachs DH, Galli C (2009) Genetic manipulation in pigs. Current Opinion in Organ Transplantation, 14, 148-153.

Scott TA, Quintaneiro LM, Norvaisas P, Lui PP, Wilson MP, Leung K-Y, Herrera-Dominguez L, Sudiwala S, Pessia A, Clayton PT, Bryson K, Velagapudi V, Mills PB, Typas A, Greene NDE, Cabreiro F (2017) Host-microbe co-metabolism dictates cancer drug efficacy in C. elegans. Cell, 169, 442-456.

Smith DG, Robinson HJ, Clark DM (1945) The influence of streptomycin and streptothricin on the intestinal flora of mice. Journal of Bacteriology, 50, 613-621.

Snel J, Heinen PP, Blok HJ, Carman RJ, Duncan AJ, Allen PC, Collins MD (1995) Comparison of 16S rRNA sequences of segmented filamentous bacteria isolated from mice, rats, and chickens and proposal of "Candidatus Arthromitus". International Journal of Systematic and Evolutionary Microbiology, 45, 780-782.

Sommer F, Bäckhed F (2013) The gut microbiota-Masters of host development and physiology. Nature Reviews Microbiology, 11, 227-238.

Stappenbeck TS, Virgin HW (2016) Accounting for reciprocal host-microbiome interactions in experimental science. Nature, 534, 191-199.

Stephens WZ, Burns AR, Stagaman K, Wong S, Rawls JF, Guillemin K, Bohannan BJM (2016) The composition of the zebrafish intestinal microbial community varies across development. The ISME Journal, 10, 644-654.

Suzuki K, Meek B, Doi Y, Muramatsu M, Chiba T, Honjo T, Fagarasan S (2004) Aberrant expansion of segmented filamentous bacteria in IgA-deficient gut. Proceedings of the National Academy of Sciences, USA, 101, 1981-1986.

Turnbaugh PJ, Ridaura VK, Faith JJ, Rey FE, Knight R, Gordon JI (2009) The effect of diet on the human gut microbiome: A metagenomic analysis in humanized gnotobiotic mice. Science Translational Medicine, 1, 6ra14. 
Valente EM, Salvi S, Ialongo T, Marongiu R, Elia AE, Caputo V, Romito L, Albanese A, Dallapiccola B, Bentivoglio AR (2004) PINK1 mutations are associated with sporadic early-onset parkinsonism. Annals of Neurology, 56, 336-341.

Verbeke KA, Boobis AR, Chiodini A, Edwards CA, Franck A, Kleerebezem M, Nauta A, Raes J, van Tol EA, Tuohy KM (2015) Towards microbial fermentation metabolites as markers for health benefits of prebiotics. Nutrition Research Reviews, 28, 42-66.

Wahlström A, Sayin SI, Marschall H-U, Bäckhed F (2016) Intestinal crosstalk between bile acids and microbiota and its impact on host metabolism. Cell Metabolism, 24, 41-50.

Wallace BD, Wang H, Lane KT, Scott JE, Orans J, Koo JS, Venkatesh M, Jobin C, Yeh L-A, Mani S, Redinbo MR (2010) Alleviating cancer drug toxicity by inhibiting a bacterial enzyme. Science, 330, 831-835.

Wang J, Jia H (2016) Metagenome-wide association studies: Fine-mining the microbiome. Nature Reviews Microbiology, 14, 508-522.

Wang S, Huang XF, Zhang P, Newell KA, Wang H, Zheng K, Yu Y (2017) Dietary teasaponin ameliorates alteration of gut microbiota and cognitive decline in diet-induced obese mice. Scientific Reports, 7, 12203.

Wiles TJ, Jemielita M, Baker RP, Schlomann BH, Logan SL, Ganz J, Melancon E, Eisen JS, Guillemin K, Parthasarathy R (2016) Host gut motility promotes competitive exclusion within a model intestinal microbiota. PLoS Biology, 14, e1002517.

Xiao L, Estellé J, Kiilerich P, Ramayo-Caldas Y, Xia Z, Feng Q, Liang S, Pedersen AØ, Kjeldsen NJ, Liu C, Maguin E, Doré J, Pons N, Le Chatelier E, Prifti E, Li J, Jia H, Liu X,
Xu X, Ehrlich SD, Madsen L, Kristiansen K, Rogel-Gaillard C, Wang J (2016) A reference gene catalogue of the pig gut microbiome. Nature Microbiology, 1, 16161.

Xiao L, Feng Q, Liang S, Sonne SB, Xia Z, Qiu X, Li X, Long H, Zhang J, Zhang D, Liu C, Fang Z, Chou J, Glanville J, Hao Q, Kotowska D, Colding C, Licht TR, Wu D, Yu J, Sung JJY, Liang Q, Li J, Jia H, Lan Z, Tremaroli V, Dworzynski P, Nielsen HB, Bäckhed F, Doré J, Le Chatelier E, Ehrlich SD, Lin JC, Arumugam M, Wang J, Madsen L, Kristiansen K (2015) A catalog of the mouse gut metagenome. Nature Biotechnology, 33, 1103-1108.

Xie H, Guo R, Zhong H, Feng Q, Lan Z, Qin B, Ward KJ, Jackson MA, Xia Y, Chen X, Chen B, Xia H, Xu C, Li F, Xu X, Al-Aama JY, Yang H, Wang J, Kristiansen K, Wang J, Steves CJ, Bell JT, Li J, Spector TD, Jia H (2016) Shotgun metagenomics of 250 adult twins reveals genetic and environmental impacts on the gut microbiome. Cell Systems, 21, 572-584.

Zákostelská Z, Málková J, Klimešová K, Rossmann P, Hornová M, Novosádová I, Stehlíková Z, Kostovčík M, Hudcovic T, Štepánková R, Jůzlová K, Hercogová J, Tlaskalová-Hogenová H, Kverka M (2016) Intestinal microbiota promotes psoriasis-like skin inflammation by enhancing Th17 response. PLoS ONE, 11, e0159539.

Zevin AS, Hensley-McBain T, Miller C, Smith E, Langevin S, Klatt NR (2017) Antibiotic treatment disrupts bacterial communities in the colon and rectum of simian immunodeficiency virus-infected macaques. FEMS Microbiology Letters, $15,364$.

(特邀责任编委: 周欣 责任编辑: 时意专) 